\title{
Decentralized Multi-cell Beamforming with QoS Guarantees via Large System Analysis
}

\author{
Hossein Asgharimoghaddam*, Antti Tölli*, Luca Sanguinetti ${ }^{\dagger \ddagger}$, Merouane Debbah ${ }^{\ddagger \S}$ \\ ${ }^{*}$ Centre for Wireless Communications, University of Oulu, Oulu, Finland \\ ${ }^{\dagger}$ Dipartimento di Ingegneria dell'Informazione, University of Pisa, Pisa, Italy \\ ${ }^{\ddagger}$ Large Networks and System Group (LANEAS), CentraleSupélec, Université Paris-Saclay, Gif-sur-Yvette, France \\ $\S$ Mathematical and Algorithmic Sciences Lab, Huawei France, Paris, France
}

\begin{abstract}
This work focuses on the power minimization problem while ensuring target rates in the downlink of a multi-cell multi-user MIMO system wherein $L$ base stations (BSs) of $N$ antennas serve in total $K$ single-antenna user equipments. We assume that the transmit antennas at each BS are correlated and propose a decentralized solution to compute an approximation of the optimal beamforming vectors. The analysis is conducted in the asymptotic regime in which $N$ and $K$ grow large with a given ratio $K / N$. In particular, the proposed solution relies on the exchange of intercell interference terms whose large system approximations are computed at each BS using knowledge of local transmit correlation matrices and non-local pathlosses. Numerical results are used to evaluate the performance loss of the proposed solution compared to the optimal one and to investigate its accuracy in systems of finite size.
\end{abstract}

\section{INTRODUCTION}

The road forward for satisfying the increasing number of users and high rates expectations in 5G systems is very high spatial utilization. Among the different technologies in this context, massive MIMO is considered as one of the most promising [1]. Under the assumption of uncorrelated channels, if the number of antennas $N$ goes to infinity and the number of UEs $K$ is maintained fixed, the performance of massive MIMO systems become limited only by pilot contamination and simple matched filter (MF) and maximum ratio transmission (MRT) with no cooperation among cells can entirely eliminate the uplink and downlink multicell interference. However, the maximum number of antennas at each base station (BS) is limited in practice. In such a case, interference-aware precoder design with cooperation among cells should be applied for optimal handling of the remaining inter-cell interference (ICI).

Coordinated multi-cell resource allocation is generally formulated as optimization problems in which a desired network utility is maximized subject to some requirements [2], [3]. In particular, the coordinated multi-cell minimum power beamforming approach aims to satisfy a given signal-tointerference-plus-noise ratio (SINR) for all users while minimizing the total transmitted power [2]. In the last years, different centralized and decentralized solutions have been suggested to tackle this problem [2]-[4]. All these solutions require some exchange of information that depends on the actual channel conditions. This makes them unsuited for scenarios with highly varying channels or with large values of $N$ and $K$ (as envisioned in 5G networks).

A possible way out to the above issue relies on conducting the analysis in the large system regime in which $N$ and $K$ grow large with a non-trivial ratio $K / N$. In these cir- cumstances, tools from random matrix theory allow to get explicit expressions, that depend only on the large-scale fading components, for (most) performance metrics. The large system analysis of the minimum power beamforming problem was first addressed in [5] for a simple two-cell network with perfect channel state information (CSI). Some more recent results can be found in [6]-[10]. A common drawback of these works is that the target rates are not guaranteed to be achieved when $N$ is finite and relatively small. This is because the approximation errors translate into fluctuations in the resulting SINR values.

To overcome this issue, in [11] the authors consider the case of uncorrelated antennas at the BSs and extend the results in [3] to decompose the multicell beamforming problem into different subproblems (one for each BS) coupled only by the intercell interference among BSs. The large dimension approximation for the ICI terms is then used to derive an approximately optimal distributed algorithm that relies only on the exchange, among nearby BSs, of the large-scale fading components. Compared to the centralized solution, [11] requires lower backhaul exchange and processing load but a slightly higher transmit power. If the per-user correlation matrix model is used [12], then correlation matrices must be also exchanged via the backhaul links. This might be a potential problem when the dimension of the network is large.

To avoid such a potential issue, this work proposes a heuristic simplification of the algorithm presented in [12]. In particular, we assume that only partial knowledge of the non-local statistics is available at each BS. More specifically, each BS $b$ is assumed to estimate the correlation matrices for all local channels (from BS $b$ to all users) while the correlation matrices from all other BSs $i \neq b$ to all users are not known locally at the given BS $b$. Only the largescale attenuation (due to pathloss and fading) are available for the non-local channels. The latter are then sent to the corresponding BSs for computing powers and beamforming vectors so as to meet SINR constraints. Numerical results show that the penalty from using partial non-local statistics is slightly higher transmission power than in [12].

\section{SySTEM MODEL}

Consider the downlink of a multi-cell multi-user MIMO system composed of $L$ cells where each BS has $N$ antennas. A total number of $K$ single-antenna UEs are randomly dropped in the coverage area. We assume that each UE is attached to a single BS while receiving interfering data from other BSs. We call $\mathcal{U}_{b}$ the set of UEs served by BS $b$ and denote $b_{k}$ the index 
of the BS associated to UE $k$. The set of all UEs is denoted by $\mathcal{U}$ whereas $\mathcal{B}$ collects all $\mathrm{BS}$ indexes. Under this convention, we define $\mathbf{h}_{b, k} \in \mathbb{C}^{N}$ as the channel from BS $b$ to UE $k$. The per-user channel correlation model is adopted [13]. Then, we have that $\mathbf{h}_{b, k}=\boldsymbol{\Theta}_{b, k}^{1 / 2} \mathbf{z}_{b, k}$ where $\mathbf{z}_{b, k} \sim \mathcal{C N}\left(\mathbf{0}, 1 / N \mathbf{I}_{N}\right)$ is the small-scale fading channel and $\Theta_{b, k} \in \mathbb{C}^{N \times N}$ accounts for the correlation among antennas at the BS. The matrices $\Theta_{b, k}$ are assumed to change slowly compared to the channel coherence time. The above per-user channel correlation model is quite general and allows to model different propagation environments that naturally arise in cellular networks [13]. In the simple i.i.d case, the correlation matrix $\Theta_{b, k}$ reduces to $a_{b, k} \mathbf{I}_{N}$ where $a_{b, k}$ is the large-scale attenuation from BS $b$ to UE $k$ due to pathloss and shadowing.

Denoting by $\mathbf{w}_{k} \in \mathbb{C}^{N}$ the precoding vector of UE $k$ from its intended BS, the received signal can be written as

$$
\begin{aligned}
y_{b_{k}, k}=\mathbf{h}_{b_{k}, k}^{H} \mathbf{w}_{k} s_{k} & +\sum_{i \in \mathcal{U}_{b_{k}} \backslash k} \mathbf{h}_{b_{k}, k}^{H} \mathbf{w}_{i} s_{i}+ \\
& +\sum_{b \in \mathcal{B} \backslash b_{k}} \sum_{l \in \mathcal{U}_{b}} \mathbf{h}_{b, k}^{H} \mathbf{w}_{l} s_{l}+n_{k}
\end{aligned}
$$

where $s_{i}$ is the symbol transmitted to UE $i$ with $\mathrm{E}\left\{\left|s_{i}\right|^{2}\right\}=1$, and $n_{k}$ is the thermal noise that follows $\sim \mathcal{C N}\left(0, \sigma^{2}\right)$.

\section{PRoblem Formulation}

The optimization problem for attaining the optimal beamforming vectors $\left\{\mathbf{w}_{k}\right\}$ as proposed by [3] can be stated as

$$
\begin{array}{ll}
\min _{\left\{\mathbf{w}_{k}\right\},\left\{\epsilon_{b, k}\right\}} & \sum_{k=1}^{K} \mu_{k}\left\|\mathbf{w}_{k}\right\|^{2} \\
\text { subject to } & \operatorname{SINR}_{k} \geq \gamma_{k} \quad \forall k \\
& \sum_{l \in \mathcal{U}_{b}}\left|\mathbf{h}_{b, k}^{H} \mathbf{w}_{l}\right|^{2} \leq \epsilon_{b, k} \quad \forall b, \forall k \notin \mathcal{U}_{b}
\end{array}
$$

where the SINR takes the form

$$
\operatorname{SINR}_{j k}=\frac{\left|\mathbf{h}_{b_{k}, k}^{H} \mathbf{w}_{k}\right|^{2}}{\sum_{i \in \mathcal{U}_{b_{k}} \backslash k}\left|\mathbf{h}_{b_{k}, k}^{H} \mathbf{w}_{i}\right|^{2}+\sum_{b \in \mathcal{B} \backslash b_{k}} \epsilon_{b, k}+\sigma^{2}}
$$

and $\epsilon_{b, k}$ accounts for the interference experienced by UE $k$ from BS $b$. Also, $\left\{\gamma_{k}\right\}$ represent the target SINRs whereas the weights $\left\{\mu_{k}\right\}$ reflect the different power budget at each BS. As mentioned in the Introduction, the above problem can be solved using different approaches based for example on centralized SOCP formulation [3] and standard dual or primal decomposition techniques [3], [14]. Next, we first revise the uplink-downlink duality approach and then provide the main results from its large system analysis that will be instrumental to derive a distributed beamforming algorithm.

\section{A. Solution via uplink-downlink duality}

Using the uplink-downlink duality [2], the optimal $\mathbf{w}_{k}$ is found to be $\mathbf{w}_{k}=\sqrt{\delta_{k}} \mathbf{v}_{k}$ with

$$
\mathbf{v}_{k}=\left(\sum_{l \in \mathcal{U} \backslash k} \lambda_{l} \mathbf{h}_{b_{k}, l} \mathbf{h}_{b_{k}, l}^{H}+\mu_{k} \mathbf{I}_{N}\right)^{-1} \mathbf{h}_{b_{k}, k}
$$

where $\left\{\lambda_{k}\right\}$ correspond to the dual uplink powers and $\left\{\delta_{k}\right\}$ are weighting coefficients from which the downlink powers $\left\{p_{k}\right\}$ are obtained as $p_{k}=\delta_{k}\left\|\mathbf{v}_{k}\right\|^{2}$. The values of $\left\{\delta_{k}\right\}$ must be computed as

$$
\boldsymbol{\delta}=\sigma^{2} \mathbf{G}^{-1} \mathbf{1}_{K}
$$

where the $(i, j)$ th element of $\mathbf{G} \in \mathbb{C}^{K \times K}$ is

$$
[\mathbf{G}]_{i, k}= \begin{cases}\frac{1}{\gamma_{k}}\left|\mathbf{h}_{b_{k}, k}^{H} \mathbf{v}_{k}\right|^{2} & \text { for } i=k \\ -\left|\mathbf{h}_{b_{k}, i}^{H} \mathbf{v}_{k}\right|^{2} & \text { for } i \neq k .\end{cases}
$$

The dual uplink powers $\left\{\lambda_{k}\right\}$ can be calculated iteratively as follows

$$
\lambda_{k}(t+1)=\lambda_{k}(t) \frac{\gamma_{k}}{\Gamma_{k}(t)}
$$

where $t$ is the iteration index and $\Gamma_{k}(t)$ is given by

$$
\Gamma_{k}(t)=\lambda_{k}(t) \mathbf{h}_{b_{k}, k}^{H}\left(\sum_{l \in \mathcal{U} \backslash k} \lambda_{l}(t) \mathbf{h}_{b_{k}, l} \mathbf{h}_{b_{k}, l}^{H}+\mu_{k} \mathbf{I}_{N}\right)^{-1} \mathbf{h}_{b_{k}, k}
$$

which corresponds to the SINR at iteration $t$ for the set of uplink powers $\left\{\lambda_{k}(t)\right\}$. The above set of equations defines an algorithm, which gives the optimal precoding vectors $\left\{\mathbf{w}_{k}\right\}$. However, the evaluation of (5) requires a global channel state information, which makes its distributed implementation a difficult task, especially when $N$ and $K$ are large.

\section{B. Solution via large system analysis}

To overcome the above issues, we assume that $N, K \rightarrow$ $\infty$ with $K / N \in(0, L)$ and use the large system analysis to compute the so-called deterministic equivalents of $\left\{\lambda_{k}\right\}$ and $\left\{\delta_{k}\right\}$. For technical reasons [13], the following assumption is made.

Assumption 1. The spectral norm of $\left\{\boldsymbol{\Theta}_{b, k}\right\}$ is uniformly bounded:

$$
\limsup _{N, K \rightarrow \infty} \max _{\forall b, k}\left\{\left\|\boldsymbol{\Theta}_{b, k}\right\|\right\}<\infty .
$$

Then, the following result is found [15].

Theorem 1. Let Assumption 1 hold. If $N, K \rightarrow \infty$ with $K / N \in(0, L)$, then $\lambda_{k}-\bar{\lambda}_{k} \rightarrow 0$ almost surely where $\bar{\lambda}_{k}$ is given by

$$
\bar{\lambda}_{k}=\frac{\gamma_{k}}{e_{b_{k}, k}}
$$

and can be computed through the following recursive procedure. Let $t$ be the iteration index, then $\lim _{t \rightarrow \infty} \bar{\lambda}_{k}(t)=\bar{\lambda}_{k}$ and $\lim _{t \rightarrow \infty} e_{b, k}(t)=e_{b, k}$ where

$$
\bar{\lambda}_{k}(t+1)=\bar{\lambda}_{k}(t) \frac{\gamma_{k}}{e_{b_{k}, k}(t)}
$$


with $\left\{e_{b_{k}, i}(t)\right\}$ being obtained as the unique nonnegative solution of the following system of equations $\forall i$ :

$e_{b_{k}, i}(t)=\frac{\bar{\lambda}_{i}(t)}{N} \operatorname{Tr}\left(\boldsymbol{\Theta}_{b_{k}, i}\left(\frac{1}{N} \sum_{l \in \mathcal{U}} \frac{\bar{\lambda}_{l}(t) \boldsymbol{\Theta}_{b_{k}, l}}{1+e_{b_{k}, l}(t)}+\mu_{k} \mathbf{I}_{N}\right)^{-1}\right)$.

Then, we have the following result [15].

Theorem 2. Let Assumption 1 hold. If $N, K \rightarrow \infty$ with $K / N \in(0, L)$, then $[\mathbf{G}]_{i, k}-[\overline{\mathbf{G}}]_{i, k} \rightarrow 0$ almost surely with

$$
[\overline{\mathbf{G}}]_{i, k}= \begin{cases}\frac{\gamma_{k}}{\left(\overline{\left.\lambda_{k}\right)^{2}}\right.} & \text { for } i=k \\ -\frac{1}{N} \frac{e_{b_{k}, i}^{\prime}}{\bar{\lambda}_{k}\left(1+e_{b_{k}, i}\right)^{2}} & \text { for } i \neq k\end{cases}
$$

where $\left\{\bar{\lambda}_{i}\right\}$ and $\left\{e_{b, i}\right\}$ are given by Theorem 1. The vector $\mathbf{e}_{b_{k}}^{\prime}$ is computed as $\mathbf{e}_{b_{k}}^{\prime}=\left(\mathbf{I}_{N}-\mathbf{L}_{b_{k}}\right)^{-1} \mathbf{u}_{b_{k}}$ where

$$
\left[\mathbf{L}_{b_{k}}\right]_{j, i}=\frac{\bar{\lambda}_{j} \bar{\lambda}_{i}}{N^{2}} \frac{\operatorname{tr}\left(\boldsymbol{\Theta}_{b_{k}, j} \mathbf{T}_{b_{k}} \boldsymbol{\Theta}_{b_{k}, i} \mathbf{T}_{b_{k}}\right)}{\left(1+e_{b_{k}, i}\right)^{2}}
$$

and

$$
\begin{aligned}
\mathbf{u}_{b_{k}}=\left[\frac{\bar{\lambda}_{1}}{N} \operatorname{tr}\left(\boldsymbol{\Theta}_{b_{k}, 1} \mathbf{T}_{b_{k}} \boldsymbol{\Theta}_{b_{k}, k} \mathbf{T}_{b_{k}}\right), \ldots,\right. \\
\left.\frac{\bar{\lambda}_{K}}{N} \operatorname{tr}\left(\boldsymbol{\Theta}_{b_{k}, K} \mathbf{T}_{b_{k}} \boldsymbol{\Theta}_{b_{k}, k} \mathbf{T}_{b_{k}}\right)\right]^{T}
\end{aligned}
$$

with $\mathbf{T}_{b}$ being the solution of

$$
\mathbf{T}_{b}=\left(\frac{1}{N} \sum_{l \in \mathcal{U}} \frac{\bar{\lambda}_{l} \boldsymbol{\Theta}_{b, l}}{1+e_{b, l}}+\mu_{b} \mathbf{I}_{N}\right)^{-1} .
$$

A known problem with any algorithm relying only on the asymptotic analysis is that the approximation errors translate into fluctuations in the resulting SINR values. Therefore, the target rates are not guaranteed to be achieved when $N$ is finite and relatively small. This issue is addressed in the next section.

\section{Distributed Optimization}

We begin by observing that any fixed ICI term in (2) results in a suboptimal solution that, however, always satisfies the SINR constraints. The price to pay, compared to the optimum, is an increase of the transmit power [11], [12]. Next, we look for a possible solution providing a good approximation of the ICI values by means of a distributed algorithm.

Note that the ICI from BS $b$ to UE $k$ can be rewritten as

$$
\epsilon_{b, k}=\sum_{l \in \mathcal{U}_{b}}\left|\mathbf{h}_{b, k}^{H} \mathbf{w}_{l}\right|^{2}=\sum_{l \in \mathcal{U}_{b}} \delta_{l}\left|\mathbf{h}_{b, k}^{H} \mathbf{v}_{l}\right|^{2} .
$$

An approximation of the coefficients $\left\{\delta_{l}\right\}$ can be computed as

$$
\overline{\boldsymbol{\delta}}=\sigma^{2} \overline{\mathbf{G}}^{-1} \mathbf{1}_{K}
$$

where $\overline{\mathbf{G}}$ is obtained through Theorem 2. The terms $\left|\mathbf{h}_{b, k}^{H} \mathbf{v}_{l}\right|^{2}$ in (16) correspond to the non-diagonal elements of $\mathbf{G}$. Therefore, $\epsilon_{b, k}$ can be reasonably approximated as

$$
\epsilon_{b, k} \approx-\sum_{l \in \mathcal{U}_{b}} \bar{\delta}_{l}[\overline{\mathbf{G}}]_{k, l}
$$

A close inspection of Theorems 1 and 2 reveals that the computation of $\left\{\bar{\lambda}_{l}\right\}$ and $\overline{\mathbf{G}}$ requires knowledge of $\left\{\boldsymbol{\Theta}_{b, k}\right\}$. Although each $\boldsymbol{\Theta}_{b, k}$ changes slowly in time compared to small-scale fading components and can be usually represented by simple mathematical models (depending on a small number of system parameters), the exchange of such information among coupled BSs via backhaul links may not be practical when the network size becomes large.

To overcome this potential issue, we propose to compute an approximation of the ICI values using full knowledge of local statistics and only partial knowledge of the nonlocal ones. More specifically, we assume that each BS $b$ is able to estimate (perfectly) the channel correlation matrices $\boldsymbol{\Theta}_{b, k} \forall k$ while the correlation matrices $\boldsymbol{\Theta}_{i, k} \forall k$ from all other BSs $i \neq b$ are not known locally at BS $b$. Only the largescale attenuation (due to pathloss and fading) is available for the non-local channels. The first assumption relies on the observation that the correlation matrix remains constant for a sufficiently large number of reception phases to be accurately estimated at the BS. The second one is motivated by the observation that most current standards require the users to periodically report Received Signal Strength Indication (RSSI) values to their serving BSs (usually using orthogonal uplink resources). Under the assumption that nearby BSs are also able to receive such RSSI measurements, a partial knowledge of the non-local channel statistics can be obtained without any information exchange through backhaul links.

Under the above assumptions, each BS can locally compute (through Theorems 1 and 2) approximations for $\left\{\bar{\lambda}_{l}\right\}$ and $\overline{\mathbf{G}}$. The latter can thus be used in (18) to locally obtain ICI values. Finally, each BS must send the UE specific ICI values to the respective BSs. The solution is summarized in Algorithm 1.

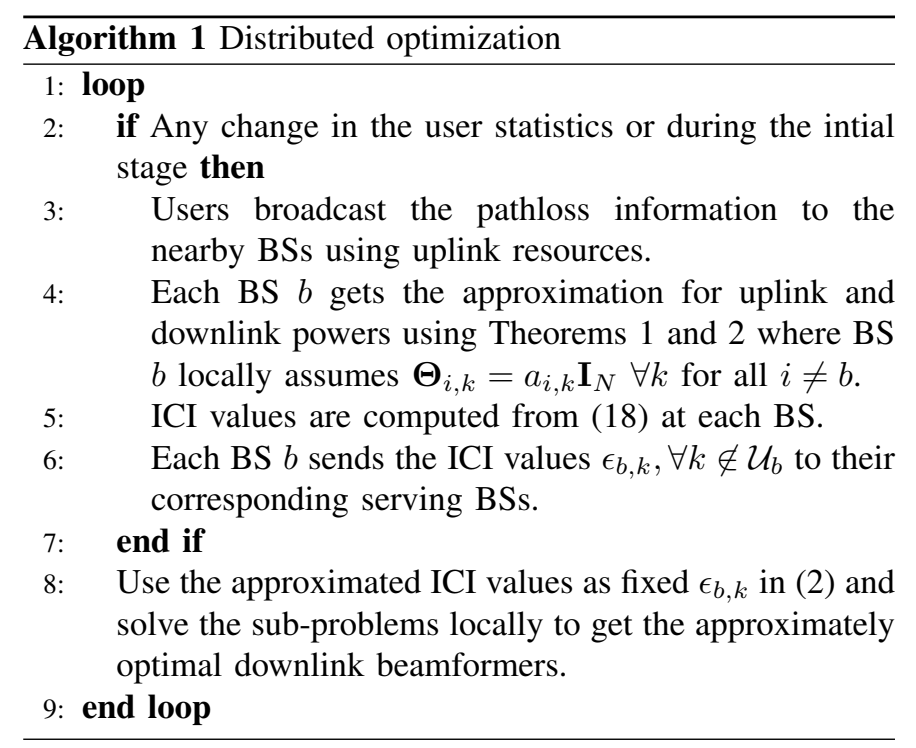

The exchange rate of Algorithm 1 results to be significantly lower than that required by [12]. This is because only some UE specific scalars (ICI values) must be sent to their serving BSs at each update interval while correlation matrices must be exchanged in [12]. 


\section{NumericAl ANALYSis}

Monte Carlo simulations are now used to validate the performance of the proposed solution. We consider a network with $L=7$ cells and assume that UEs are equally distributed among cells. The pathloss function is modelled as $a_{b, k}=\left(d_{0} / d_{b, k}\right)^{2.5}$ where $d_{b, k}$ represents the distance between BS $b$ and UE $k$ and $d_{0}=1 \mathrm{~m}$ is the reference distance. The pathloss from a generic BS to the boundary of the reference distance of the neighboring $\mathrm{BS}$ is fixed to $75 \mathrm{~dB}$ (corresponding to a $1000 \mathrm{~m}$ distance between BSs). The correlation among channel entries is modeled by assuming a diffuse 2-D field of isotropic scatterers around the receivers [13], [16]. The waves arrive from an angular spread from $\varphi_{\min }$ to $\varphi_{\max }$. The correlation matrix for an antenna element spacing of $\Delta$ is given by [16]:

$$
\left[\Theta_{b, k}\right]_{j, l}=\frac{1}{\varphi_{b, k}^{\max }-\varphi_{b, k}^{\min }} \int_{\varphi_{b, k}^{\min }}^{\varphi_{b, k}^{\max }} e^{i \frac{2 \pi}{w} \Delta|j-l| \cos (\varphi)} \mathrm{d} \varphi
$$

where $w$ is the wavelength and the antenna element spacing is fixed to half the wavelength $\Delta=1 / 2 w$. In order to study the performance of the algorithm for both rank-deficient and well-conditioned matrices, we choose $\varphi_{b, k}^{\min }=-\pi$ and assign the $\varphi_{b, k}^{\max }$ based on the position of UE $k$ with respect to BS $b$. This results in various angular spreads for UEs depending on their positions.

Fig. 1 shows the transmit $\mathrm{SNR}$ in $\mathrm{dB}$ (defined as the transmit power normalized to the noise variance $\sigma^{2}$ ) for the proposed algorithm labeled as "partial statistical information" when $K / N=1 / 2$ and $\gamma_{k}=0 \mathrm{~dB} \forall k$. Comparisons are made with the simple ZF beamforming, the optimal centralized solution [3] and the decentralized solution with full statistical information [12]. Looking at (11), it can be seen that $e_{b_{k}, k}(t)$ (and thus $\lambda_{k}$ ) is directly linked to local statistics while it depends on non-local statistics via non-local uplink powers. Thus, providing an approximation for non-local uplink powers based on pathloss information, we can get a solution close to the case with full statistical information as shown in Fig. 1. However, ignoring the local statistics and relying only on the exchanged pathloss information gives a fully i.i.d decentralized solution subject to at least $2 \mathrm{~dB}$ performance loss as depicted in Fig. 1 . In this case the ICI values calculated at all BSs are the same globally, thus, there is no need to exchange the ICI values on the backhaul.

\section{CONCLUSION}

This work focused on the power minimization problem in multicell MU-MIMO networks and proposed a heuristic distributed beamforming algorithm that inherits the benefits of [12] without requiring the exchange of correlation matrices. Numerical results indicated that the proposed solution achieves the same performance as the case in which correlation matrices are exchanged. This is achieved even for small system dimensions with a reasonable performance loss with respect to the optimal one.

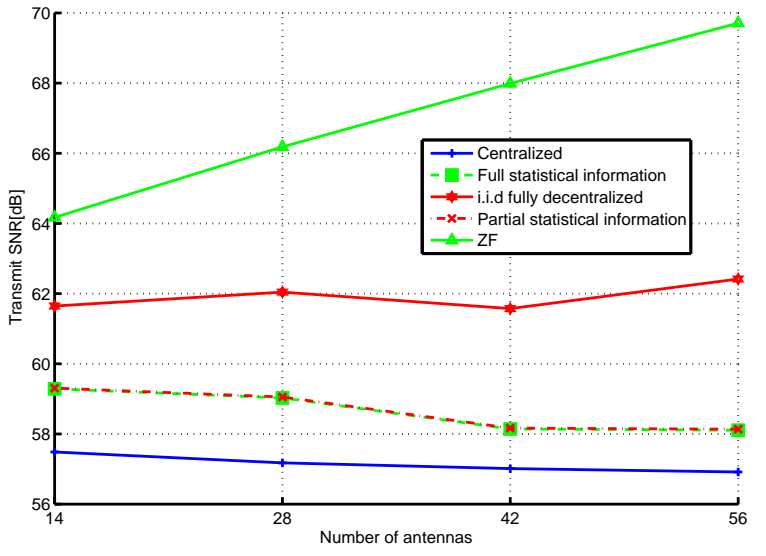

Fig. 1. Transmit SNR vs. number of antennas with $\frac{K}{N}=\frac{1}{2}$ and $\gamma=0 \mathrm{~dB}$. REFERENCES

[1] J. Hoydis, S. ten Brink, and M. Debbah, "Massive MIMO in the UL/DL of cellular networks: How many antennas do we need?" IEEE Journal on selected Areas in Communications, vol. 31, no. 2, February 2013.

[2] H. Dahrouj and W. Yu, "Coordinated beamforming for the multicell multi-antenna wireless system," IEEE Trans. Wireless Commun., vol. 9, no. 5, pp. 1748-1759, May 2010.

[3] A. Tölli, H. Pennanen, and P. Komulainen, "Decentralized minimum power multi-cell beamforming with limited backhaul signaling," IEEE Transactions on Wireless Communications, vol. 10, no. 2, Feb. 2011.

[4] H. Pennanen, A. Tolli, and M. Latva-aho, "Decentralized coordinated downlink beamforming via primal decomposition," IEEE Signal Process. Lett., vol. 18, no. 11, Nov 2011.

[5] R. Zakhour and S. Hanly, "Base station cooperation on the downlink: Large system analysis," IEEE Trans. Inf. Theory, vol. 58, no. 4, Apr. 2012.

[6] L. Sanguinetti, E. Bjornson, M. Debbah, and A. Moustakas, "Optimal linear precoding in multi-user MIMO systems: A large system analysis," in IEEE Global Communications Conference (GLOBECOM), Dec 2014.

[7] L. Sanguinetti, A. Moustakas, E. Bjornson, and M. Debbah, "Large system analysis of the energy consumption distribution in multi-user MIMO systems with mobility," IEEE Trans. Wireless Commun., vol. 14, no. 3, pp. 1730 - 1745, March 2015 .

[8] L. Sanguinetti, R. Couillet, and M. Debbah, "Large system analysis of base station cooperation for power minimization," IEEE Trans. Wireless Commun., Sept. 2015, submitted. [Online]. Available: http://arxiv.org/abs/1509.00731

[9] S. Lakshminaryana, J. Hoydis, M. Debbah, and M. Assaad, "Asymptotic analysis of distributed multi-cell beamforming," in IEEE 21st International Symposium on Personal Indoor and Mobile Radio Communications (PIMRC), Sept 2010, pp. 2105-2110.

[10] S. Lakshminaryana, M. Assaad, and M. Debbah, "Coordinated multi-cell beamforming for massive MIMO: A random matrix approach," IEEE Trans. Inf. Theory, vol. 61, no. 6, 2015.

[11] H. Asgharimoghaddam, A. Tölli, and N. Rajatheva, "Decentralizing the optimal multi-cell beamforming via large system analysis," in Proc. IEEE Int. Conf. Commun., Sydney, Australia, Jun. 2014.

[12] H. Asgharimoghaddam, A. Tolli, and N. Rajatheva, "Decentralized multi-cell beamforming via large system analysis in correlated channels," in Signal Processing Conference (EUSIPCO), 2013 Proceedings of the 22nd European, 2014, pp. 341-345.

[13] S. Wagner, R. Couillet, M. Debbah, and D. Slock, "Large system analysis of linear precoding in correlated MISO broadcast channels under limited feedback," IEEE Trans. Inf. Theory, vol. 58, no. 7, pp. 4509-4537, July 2012.

[14] H. Pennanen, A. Tolli, and M. Latva-aho, "Multi-cell beamforming with decentralized coordination in cognitive and cellular networks," IEEE Transactions on Signal Processing, vol. 62, no. 2, pp. 295-308, 2014.

[15] H. Asgharimoghaddam, A. Tölli, and N. Rajatheva, "Analysis of minimum power beamforming based on theory of large-dimension random matrices," University of Oulu, Tech. Rep., May 2014. [Online]. Available: http://www.ee.oulu.fi/ atolli/Eusipco2014SupportingDocument.pdf

[16] W. C. Jakes, Microwave Mobile Communications, 1st ed. Wiley-IEEE Press, 1994 